\title{
Endoscopic Resection of a Colonic Lipoma Under Endoscopic Ultrasound Guidance
}

Recent trials have examined the usefulness of endoscopic resection in the treatment of colonic submucosal tumors, as an alternative to invasive surgery $(1-4)$. Perforation attributed to snaring of the muscularis propria during endoscopic removal has also been reported (2). The present report describes a case in which endoscopic resection under endoscopic ultrasound guidance was used to remove a colonic lipoma.

A 57-year-old man was admitted to hospital for examination and treatment of a submucosal tumor in the descending colon. Total colonoscopy showed a yellowish, semipedunculated polypoid lesion, with a smooth surface, in the descending colon. The lesion was relatively soft, and showed a cushion sign. A biopsy showed only normal colonic mucosa. We inserted a flexible sonographic probe through the biopsy channel of the colonoscope during direct visualization of the lesion, and carried out endoscopic ultrasonography (EUS) using an ultrasonic miniprobe (SP501, frequency $15 \mathrm{MHz}$, Fuji Photo Optical Co., Omiya, Japan). EUS demonstrated a slightly hyperechoic solid tumor in the submucosa, with no continuity with the muscularis propria layer (Figure 1). The lesion was diagnosed as a lipoma, and was considered to be resectable by endoscopic resection.
Endoscopic resection guided by the endoscopic ultrasound probe was carried out as follows. Using an endoscopic injector, about $10 \mathrm{ml}$ of concentrated glycerin solution mixed with a small volume of epinephrine was injected underneath the lesion. Adequate separation (about $8 \mathrm{~mm}$ ) of the margin of the hyperechoic tumor from the underlying muscle layer was then confirmed using EUS (Figure 2). A snare polypectomy was performed at the base of the lesion. There were no complications. Histological examination of the specimen confirmed a complete resection of the colonic lipoma. 
a, b

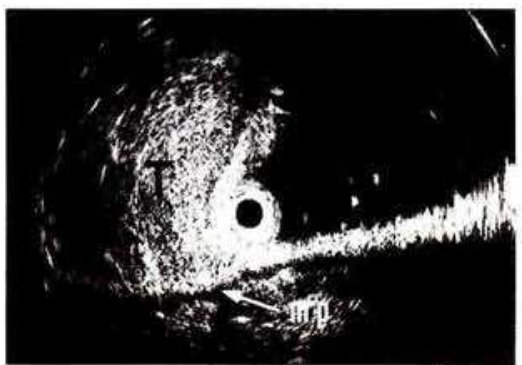

$\mathrm{a}, \mathrm{b}$

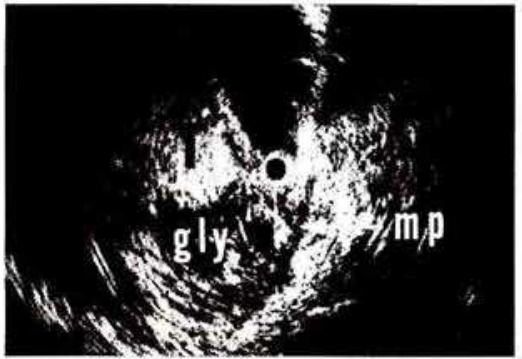

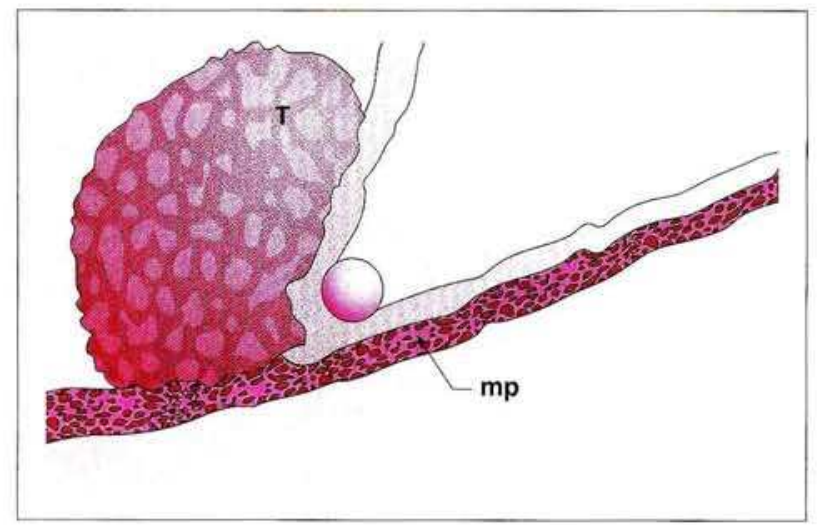

Figure 1a: EUS demonstrates a hyperechoic solid tumor in the submucosal layer. without continuity with the muscularis propria layer, b Diagram clarifying the EUS findings. mp: Muscularis propria: T: Tumor.

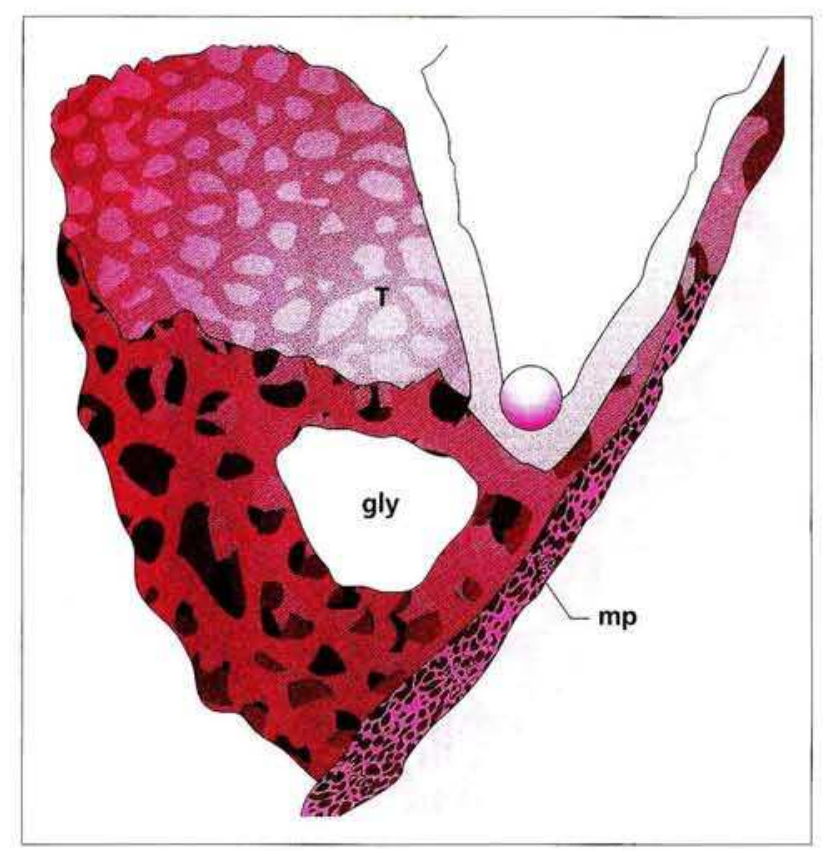

Figure 2a: EUS image of the lesion after injection of an epinephrine-supplemented glycerin solution into the submucosal layer. There is adequate separation of the margin of the hyperechoic submucosa tumor from the underlying muscle layer. b Diagram clarifying the EUS findings. gly: Glycerin solution: mp: Muscularis propria; T: Tumor.

T. Ochiai', K. Akahoshi', S. Hamada', $Y$. Chijiiwa', H. Nawata', M. Namoto 1 Third Dept. of Internal Medicine ${ }^{2}$ First Dept. of Pathology,

Faculty of Medicine, Kyushu University, Fukuoka, Japan

\section{References}

1. Yu JP, Luo HS, Wang XZ. Endoscopic treatment of submucosal lesions of the gastrointestinal tract. Endoscopy 1992; 24: $190-3$.

2. Pfeil SA, Weaver MG, Abdul-Karim FW, Yang P. Colonic lipomas: outcome of endoscopic removal. Gastrointest Endosc 1990; 36: 435-8.

3. Chang KJ, Yoshinaka R, Nguyen P. Endoscopic ultrasound-assisted band ligation: a new technique for resection of submucosal tumors. Gastrointest Endosc 1996; 44: 720-2.
4. Iwakiri Y, Akahoshi K, Hamada S, et al. Endoscopic removal of a lymphangioma of the colon: the diagnostic value of an endoscopic ultrasound probe - a case report. Endoscopy [in press].

5. Akahoshi K, Chijiiwa Y, Tanaka M, Harada N, Nawata H. Endosonography probe-guided endoscopic mucosal resection of a gastric neoplasm. Gastrointest Endosc 1995; 42: 248-52.

Corresponding Author

T. Ochiai, M.D.

Third Dept. of Internal Medicine

Faculty of Medicine

Kyushu University

Fukuoka 812-82

Japan

Fax: + 81-92-642-5287 submucosal tumors throughout the tive tract. 